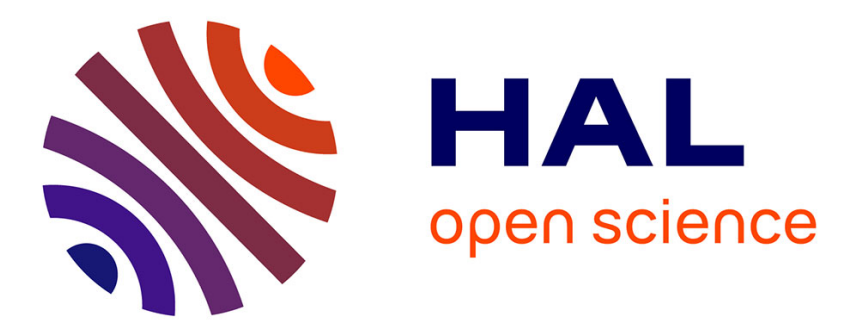

\title{
A Biological Model of Acupuncture and its Derived Mathematical Modeling and Simulations
}

\author{
Marc Thiriet, Yannick Deleuze, Tony W.H. Sheu
}

\section{To cite this version:}

Marc Thiriet, Yannick Deleuze, Tony W.H. Sheu. A Biological Model of Acupuncture and its Derived Mathematical Modeling and Simulations. Communications in Computational Physics, 2015, 18 (4), pp.831-849. 10.4208/cicp.121214.250515s . hal-01240291

\section{HAL Id: hal-01240291 \\ https://hal.sorbonne-universite.fr/hal-01240291}

Submitted on 9 Dec 2015

HAL is a multi-disciplinary open access archive for the deposit and dissemination of scientific research documents, whether they are published or not. The documents may come from teaching and research institutions in France or abroad, or from public or private research centers.
L'archive ouverte pluridisciplinaire HAL, est destinée au dépôt et à la diffusion de documents scientifiques de niveau recherche, publiés ou non, émanant des établissements d'enseignement et de recherche français ou étrangers, des laboratoires publics ou privés. 


\title{
A Biological Model of Acupuncture and its Derived Mathematical Modeling and Simulations
}

\author{
Marc Thiriet $^{1,2,3, *}$, Yannick Deleuze ${ }^{1,4}$, and Tony W.H. Sheu ${ }^{4,5}$ \\ 1 Sorbonne Universités, UPMC Univ Paris 06, UMR 7598, Laboratoire Jacques- \\ Louis Lions, F-75005, Paris, France. \\ 2 CNRS, UMR 7598, Laboratoire Jacques-Louis Lions, F-75005, Paris. \\ ${ }^{3}$ INRIA Paris-Rocquencourt, EPC REO, BP105, F-78153 Le Chesnay Cedex. \\ ${ }^{4}$ Department of Engineering Science and Ocean Engineering, National Taiwan Uni- \\ versity, Taipei, Taiwan. \\ ${ }^{5}$ Center for Advanced Study in Theoretical Sciences (CASTS), National Taiwan Uni- \\ versity, Taipei, Taiwan.
}

${ }^{*}$ Corresponding author. Email addresses: marc.thiriet@upmc.fr (M. Thiriet), yannick.deleuze@upmc.fr (Y. Deleuze), twhsheu@ntu.edu.tw (T.W.H. Sheu) 


\begin{abstract}
Aims) Acupuncture was employed since 2 millenaries, but the underlying mechanisms are not globally handled. The present study is aimed at proposing an explanation by pointing out involved processes and a convincing modeling to demonstrate its efficiency when carried out by trained practitioners.

(Method) In the absence of global knowledge of any mechanism explaining the acupuncture process, a biological model is first developed, based on stimulation in a given domain around the needle tip of a proper mastocyte population by a mechanical stress, electrical, electromagnetic, or heat field. Whatever the type of mechanical or physical stimuli, mastocytes degranulate. Released messengers either facilitate the transfer of main mediators, or target their cognate receptors of local nerve terminals or after being conveyed by blood their receptors on cerebral cells. Signaling to the brain is fast by nervous impulses and delayed by circulating messengers that nevertheless distribute preferentially in the brain region of interest due to hyperemia. The process is self-sustained due to mastocyte chemotaxis from the nearby dense microcirculatory circuit and surrounding mastocyte pools, which are inadequate for acupuncture, but serve as a signal relay. A simple mathematical model is solved analytically. Numerical simulations are also carried out using the finite element method with mesh adaptivity. (Results) The analytical solution of the simple mathematical model demonstrates the conditions filled by a mastocyte population to operate efficiently. A theorem gives the blow-up condition. This analytical solution serves for validation of numerical experiments. Numerical simulations show that when the needle is positioned in the periphery of the acupoint or outside it, the response is too weak. This explains why a long training is necessary as the needle implantation requires a precision with a magnitude of the order of $1 \mathrm{~mm}$.

(Conclusion) The acupoint must contain a highly concentrated population of mastocytes (e.g., very-high-amplitude, small-width Gaussian distribution) to get an initial proper response. Permanent signaling is provided by chemotaxis and continuous recruitment of mastocytes. Therefore, the density and distribution of mastocytes are crucial factors for efficient acupuncture as well as availability of circulating and neighboring pools of mastocytes.
\end{abstract}

AMS subject classifications: 76M10, 92C10, 92C17, 92C50

Key words: acupoint, chemotaxis, finite element method, mastocyte, mechanotransduction, neural and hormonal control

\title{
1 Introduction
}

Traditional Chinese medicine defines acupuncture points, or acupoints, for therapeutic objectives, more than 2500 years ago. Acupuncture was aimed at relieving a pathological state by liberating the sequestered energy and rearranging the balance of yin and yang to ensure homeostasis. A disease was indeed supposed to result from an imbalance between Yin and Yang. Yang and Yin are 2 fundamental opposing, complementary, and interdependent forces found in all things in the universe, with traces of one in the other, 
that support each other and can transform into one another. Nothing in the universe is completely Yin or Yang; everything is a mixture of these two. In particular, Yang may be considered as mental activity in its strength aspect, Yin mental activity in its imaginative aspect; in other words, Yang constructs, Yin instructs, or conversely. Yin is related to static and hypoactive phenomena, Yang to dynamic and hyperactive processes, or conversely.

An acupuncture needle is inserted into selected acupoints on the body's surface, on which mechanical or other types of physical stimulations are exerted (e.g., moxibustion), in particular to cause analgesia. Afferent paths are stimulated to elicit the de-qi sensation and signal to adequate zones in the central nervous system. Acupoints are transfer sites of qi. Meridians (Chinese: jing) and collaterals (Chinese: luo) are communication paths for qì, the vital energy master of body fluids, which can be transported to acupoints. These spatially restricted sites that do not correspond to a specialized biological tissue, but to localized structural and fonctional units, from which energy pours and pervades into the body's tissues.

The present study focuses on the permanent regime of acupuncture, that is, once the needle has been implanted and the stress field in the subcutaneous connective tissue is fully established. The reader is invited to read the accompanying paper [1] to get information on events occurring during the transient regime.

\section{Context.}

The brain is a complex processor that can sense chemical, physical, and mechanical signals, treat them, and transmit an extremely quick as well as delayed long-lasting output for adaptation using neural and vascular routes. Surgical interventions can be carried out using either general anesthesia, that is, a medically induced coma, or acupuncture, that is, performing tasks in conscious subjects naturally anesthetized. In the latter case, the brain that is capable of synthesizing opioids and antalgics is stimulated from acupoints that are known since 2 millenaries. In addition to a better confort for the patient who avoids coma, the cost for the health service is much lower.

\section{Acupuncture modalities.}

Acupuncture requires a long-term training to handle acupoints. Four techniques exist: (1) development of a local mechanical stress field by needle motions (lifting-thrusting cycle or rotation) at acupoints; (2) development of a local temperature field by directly applying a heating moxa (mugwort herb) stick on the skin or indirectly by applying this stick on the acupuncture needle (moxibustion) at acupoints; (3) development of a local electrical field by applying a small electric current between a pair of acupuncture needles (electroacupuncture, or percutaneous electrical nerve stimulation [PENS]) at acupoints; and (4) excitation of putative light-sensitive G-protein-coupled receptors on the surface of mastocytes by laser beam.

\section{Subcutaneous connective tissue.}

The skin consists of three main layers: the epidermis, dermis, and hypodermis. Beneath the skin, the subcutaneous connective tissue lies above skeletal muscles. It spreads throughout the body and is in continuity with interstitial connective tissues surrounding all muscles, organs, and neurovascular bundles. The connective tissue contains scattered 
cells immersed in the extracellular matrix through which the aqueous phase, or interstitial fluid, can flow [2].

Cell types of connective tissue include both resident cells, such as fibroblasts, fibrocytes, mastocytes, and macrophages, and scouting cells of the immunity (e.g., monocytes, lymphocytes, and granulocytes).

The extracellular matrix is made up of collagen and elastin fibers and a ground substance (i.e., mostly glycoproteins and proteoglycans), a hydrated medium that is more or less polymerized according to the physical environmental conditions. Collagen and elastin fibers are the main elements responsible for the matrix rheological properties. Elastin fibers enable tissue deformation up to a certain level, the energy being restituted upon mechanical stress removal. Collagen fibers yield tensile resistance.

The extracellular matrix transmits mechanical stresses to the embedded cell as well as from the cell, when the latter contracts or migrates. It then participates in mechanotransduction, that is, the conversion by any cell of mechanical stimuli sensed at its surface (plasma membrane) into chemical signaling (i.e., a cascade of chemical reactions leading to transfer in the extracellular space [exocytosis] of stored materials and gene transcription [protein synthesis]).

The subcutaneous connective tissue can be considered as a fluid-filled deformable porous medium. The Darcy and Brinkman equations describe interstitial incompressible fluid flow in the framework of a continuum. In a steady state, that is, when the subcutaneous connective tissue is permanently deformed without fluctuations of stress or strain, the matrix is in a given polymerization state and interstitial fluid is supposed to be in a new equilibrium (resting) state.

\section{Acupoint.}

Insertion into the skin of thin needles is the most common technique. An initial manual manipulation is then achieved. Any acupoint localized to the vicinity of bones, aponeuroses, muscles, and tendons that contain neural units with somatosensory receptors.

The acupoint is characterized by a high density of mastocytes. This pool of mastocytes reside close to neurovascular bundles, in a region where capillaries, lymphatic vessels, and nerve endings abound. Other features of acupoint comprise a large skin electrical conductance and high ionic concentrations (especially $\mathrm{Ca}^{++}$).

Free nerve endings and cutaneous receptors (Merkel, Meissner, Ruffini, and Pacinian corpuscles), sarcous sensory receptors (muscle spindles and tendon organs), and their afferent fibers, as well as somatic efferent fibers innervating muscles, small nerve bundles, and plexi are observed in acupoints [3]. The mechanical stress field can activate $A \alpha, A \beta$, and $\mathrm{A} \delta$ fibers, as well as $\mathrm{C}$-fibers of nervous structures at acupoints.

A nerve fiber corresponds to an axon possibly endowed with a myelin sheath, according to whether the nerve fiber is myelinated or not. Three types of peripheral nerve fibers exist according to their caliber (A-C). The A type consists of 4 subtypes of relatively large $(1-22 \mu \mathrm{m})$, myelinated fibers characterized by a high conduction velocity $(5-120 \mathrm{~m} / \mathrm{s})$ : $\mathrm{A} \alpha$ (afferent or efferent; motor and proprioceptive; bore 13-22 $\mu \mathrm{m}$; conduction velocity 
$70-120 \mathrm{~m} / \mathrm{s}$ ); $\mathrm{A} \beta$ (afferent or efferent; motor and proprioceptive; caliber 8-13 $\mu \mathrm{m}$; conduction velocity 30-90 m/s); A $\gamma$ (efferent; only motor fibers; caliber 3-7 $\mu \mathrm{m}$; conduction velocity $15-40 \mathrm{~m} / \mathrm{s}$ ); and $\mathrm{A} \delta$ (afferent; only sensory fibers [rapid pain sensation]; caliber 1-5 $\mu \mathrm{m}$; conduction velocity $5-15 \mathrm{~m} / \mathrm{s}$ ) fibers. Type-A fibers transmit impulses related to muscle, tendon, and joint movement and situation. B-type nerve fibers are also myelinated, albeit thinner $(\leq 3 \mu \mathrm{m})$. These preganglionic fibers of the autonomic nervous system transmit involuntary impulses with a slower conduction velocity $(3-15 \mathrm{~m} / \mathrm{s})$. Ctype, thin $(0.3-1.3 \mu \mathrm{m})$, unmyelinated fibers have a low conduction velocity $(0.6-2.3 \mathrm{~m} / \mathrm{s})$. They include postganglionic fibers in the autonomic nervous system as well as nerve fibers of the dorsal roots that carry sensory information (heat, pressure, and slowly pain).

\section{Mastocyte, the master cell in acupuncture.}

Other cells located at acupoints, such as neurons, macrophages, fibroblasts, and lymphocytes, can contribute to the emission of local and endocrine signals. Mastocytes that contain numerous granules serving as stores for transmitters is the major cellular species [4].

Mastocytes are activated by a mechanical stress field (mechanotransduction), heating (thermotransduction), electrical field (electrotransduction), or light (especially laser; phototransduction). Two mastocyte states are thus considered according to the location with respect to acupoint: granulated close to the stimulation source and degranulated outside this region, that is, at a distance of about $5 \mathrm{~mm}$ from the needle in the case of mechanotransduction.

All these 4 techniques share the same mechanism, as they rely on calcium signaling. Nevertheless, mechanotransduction corresponds to the most studied operating mode, experimentally or numerically. Needle manipulation in acupuncture can cause degranulation of mastocytes upon exposure to mechanical stress [7-9].

Calcium, the primary intracellular messenger in acupuncture.

Whatever the operation mode, calcium very rapidly enters the mastocyte through proper gated $\mathrm{Ca}^{2+}$ channels in the plasma membrane. According to the operating mode, the target $\mathrm{Ca}^{2+}$ channel is mechanosensitive, thermosensitive, voltage-gated, and photosensitive. In the last-mentioned case, the $\mathrm{Ca}^{2+}$ channel may be supposed to be intrinsically photosensitive or be linked to a photosensitive G-protein-coupled receptor. It then triggers exocytosis of granules serving as stores of messengers very rapidly along cytoskeletal tracks.

Release of autacoids and messengers for remote targets.

Calcium influx in the mastocyte cytosol provokes degranulation and release of chemoattractants, neural stimulants, vasoactive agents, and endocrine substances. Therefore, acupuncture effects result from a set of signals sent from activated mastocytes at given acupoints to local nerve endings, lymphatics, and capillaries, and from these two lastmentioned structures to the heart and brain to improve the blood circulation at selected acupoints and trigger the cerebral response, respectively.

Numerous types of molecules freed by mastocytes include calcitonin gene-related peptide (CGRP), heparin, histamine, leukotrienes $\left(\mathrm{LTb}_{4}, \mathrm{LTc}_{4}, \mathrm{LTd}_{4}\right.$, and $\left.\mathrm{LTe}_{4}\right)$, platelet- 
Table 1: Signaling mediators released at acupoints (Sources: [5, 6]; CGRP: calcitonin gene-related peptide; MOR: $\mu$-opioid receptor). Mechanical stresses can activate $A \alpha, A \beta$, and $A \delta$ fibers, as well as $C$-fibers of nervous structures at acupoints and augment locally the vascular permeability to accelerate the transfer of mediators to the flowing blood.

\begin{tabular}{|c|c|c|}
\hline \multirow{2}{*}{\multicolumn{3}{|c|}{ Releasing cells }} \\
\hline & Releasing cells & Receptors \\
\hline & & Effects \\
\hline Acetylcholine & Neuron, keratinocyte & $\mathrm{M}_{2}$ \\
\hline Adenosine & & $\mathrm{A}_{1}$ \\
\hline ATP & Epidermal cells & $\mathrm{P} 2 \mathrm{X}, \mathrm{P} 2 \mathrm{Y}$ \\
\hline Bradykinin & Local cells & $\mathrm{B}_{1}, \mathrm{~B}_{2}$ \\
\hline CGRP & $\begin{array}{l}\text { Epidermal cells, T cell, } \\
\text { macrophage }\end{array}$ & \\
\hline \multicolumn{3}{|c|}{ Cytokines (interleukins, tumor-necrosis factor- $\alpha$} \\
\hline $\begin{array}{l}\text { IL1 } \beta / 6 / 8, \\
\operatorname{TNF} \alpha\end{array}$ & Local cells & $\begin{array}{l}\text { Enhanced excitability } \\
\text { of afferent fibers }\end{array}$ \\
\hline IL4/10 & Local cells & $\begin{array}{l}\text { Inhibition of inflammatory } \\
\text { signals in afferent terminals }\end{array}$ \\
\hline$\beta$-endorphin & $\begin{array}{l}\text { Fibroblasts,leukocytes, } \\
\text { keratinocyte, melanocyte }\end{array}$ & MOR \\
\hline Histamine & Mastocyte & $\mathrm{H}_{1}, \mathrm{H}_{3}$ \\
\hline GABA & Macrophage, lymphocyte & $\mathrm{GABA}_{\mathrm{A}}$ \\
\hline Glutamate & Macrophage, epidermal cells & \\
\hline Nitric oxide & Many cell types & $\begin{array}{l}\text { Inhibition of substance-P } \\
\text { release from nerve terminals } \\
\text { Stimulation of acetylcholine } \\
\text { and } \beta \text {-endorphin secretion }\end{array}$ \\
\hline Noradrenaline & Sympathetic nerve & $\alpha 2 \mathrm{AR}$ \\
\hline Serotonin & Mastocyte, platelet, & $\begin{array}{l}5 \mathrm{HT}_{1}, \\
5 \mathrm{HT}_{3} \text { (afferent nerve) }\end{array}$ \\
\hline Somatostatin & Merkel cell, keratinocyte & SstR \\
\hline Substance-P & $\begin{array}{l}\text { Mastocyte, fibroblast, } \\
\text { platelet, macrophage, } \\
\text { keratinocyte }\end{array}$ & \\
\hline Prostaglandins & Local cells & EP \\
\hline
\end{tabular}

activating factor, prostaglandin-E2, serotonin, substance-P, and thromboxane-A2 (Table 2). Mastocytes also secrete peptidases (e.g., tryptase), growth factors (e.g., FGF, CSF2, and NGF), and cytokines (e.g., interleukins and tumor-necrosis factor). Nerve endings are stimulated and release substance-P that further activates mastocytes and triggers the production of nitric oxide, a gaseous vasodilator.

Nerves and mastocytes exchange chemical messengers such as substance-P. The latter stimulates histamine and nitric oxide (NO) release. Calcitonin gene-related peptide 
Table 2: Released molecules by the mastocyte and their effects.

\begin{tabular}{|c|c|}
\hline Agent & Effects \\
\hline$\overline{\text { CGRP }}$ & $\begin{array}{l}\text { Vasodilation, } \\
\text { positive chronotropy, inotropy, and lusitropy, } \\
\text { mastocyte degranulation }\end{array}$ \\
\hline Heparin & Blood clot prevention \\
\hline Histamine & $\begin{array}{l}\text { Vasodilation (directly and via NO), } \\
\text { nerve stimulation }\end{array}$ \\
\hline Leukotrienes & Vasodilation, vascular permeability elevation \\
\hline IL, NGF, TNF & Chemotaxis \\
\hline Prostaglandin-D2 & Nerve stimulation \\
\hline Prostaglandin-E2 & $\begin{array}{l}\text { Vasodilation, } \\
\text { inhibition of mediator release }\end{array}$ \\
\hline Serotonin & Vasoconstriction followed by NO-mediated vasodilation \\
\hline Thromboxane-A2 & Vasoconstriction, platelet aggregation \\
\hline Tryptase, chymase & Matrix degradation for enhanced cell migration \\
\hline
\end{tabular}

(CGRP) and NO cause vasodilation. Nitric oxide cooperates with CGRP to increase its positive inotropic effect that raises the local blood flow in dilated vessels. Histamine is quickly catabolized, thereby acting near the site of release. Resulting vasodilation and increased vessel wall permeability support transfer of chemical mediators into the blood circulation. Serotonin has a biphasic effect, as it triggers a vasoconstriction and promotes NO release, hence subsequently vasodilation. Nerve growth factor (NGF), tumornecrosis factor (TNF), and interleukins (IL) are potent mastocyte chemoattractants. Mastocyte chemotaxis is supported by matrix degradation by secreted peptidases.

This traditional procedure of Chinese medicine relies on intra-, auto-, juxta-, para-, and endocrine signaling aimed at triggering mastocyte chemotaxis and sending messages via: (1) nerves for an immediate $(\mathcal{O}[1 \mathrm{~s}-1 \mathrm{mn}])$, fast, and transient response of the central nervous system responsible for hyperemia in a given local region of the brain, in which neurons then secrete endocannabinoids, enkephalins, endomorphins, dynorphins, and other analgesic substances, in particular, as well as a permanent response ensured by the continuous flux of activators; and (2) blood and lymph vessels for a delayed and slower reaction based on transmission of substances that are conveyed throughout the brain, but preferentially to highly perfused regions. Target nervous centers then reply by regulating the behavior of proper peripheral organs.

\section{A prolonged process.}

The process is sustained by recruitment of mastocytes (chemotaxis). The time required for the synthesis of chemical mediators in degranulated mastocytes is indeed finite with a magnitude order of one hour. Therefore, a continuous secretion of messengers relies on a permanent arrival of mastocytes into the stimulated region, where they become activated and degranulate. Two sources of incoming mastocytes include the lo- 
cal dense capillary network (circulating mastocytes) and nearby pools, that is, clusters of mastocytes situated relatively close to the stimulated region, but that cannot be used as acupoints, as their distribution does not match that at acupoint characterized by a very concentrated mastocyte population, which enables a Dirac-like response.

\section{Mechanotransduction.}

Acupoint is characterized by a cluster of mastocytes and other isolated cell types. Cells are immersed in a medium containing extracellular fluid (i.e., water and small molecules and ions) and macromolecules. Among these macromolecules, some form fibers (elastin and collagen), whereas others form hydrated meshwork of glycoproteins and proteoglycans.

Once it is subjected to a stress field, this biological medium deforms. The meshwork constituted by hydrated proteoglycans and glycoproteins can be degraded, hence undergoing fluidization. Elastin and collagen fibers are stretched and bent. They may also depolymerize, hence contributing to the medium fluidization.

After the transient regime, when the matrix reaches a new equilibrium state, that is, in the permanent regime before the following needle manipulation, proteoglycans and glycoproteins as well as fibers can repolymerize (gelification).

In the transient regime, that is, during needle manipulation and shortly after its end, the induced motion of the extracellular fluid is very slow. Moreover, convection is still hindered by fluidizing macromolecular complexes (mainly fibers and proteoglycans). When needling ceases, the fluid motion soon stops and rapid regelification hampers any further displacement. Due to the transient fluidization, convection linked to the motion of the extracellular fluid is a secondary phenomenon that can be neglected.

The plasma membrane of embedded cells makes a virtual boundary between the extra- and intracellular media. They indeed contain proteins such as integrins and adhesion plaques that define a continuum between cell and matrix constituents. Therefore, any stress imposed by a cell to its environment, or conversely by the extracellular milieu to the cell, is transmitted by these membrane structures. In the absence of organized cellular architecture such as in epithelia (e.g., the epidermis), needling disturbs mainly the extracellular milieu, inducing a local mechanical stress field that is transmitted to immersed cells. Inside the cell, the cytoskeleton also undergoes polymerization-depolymerization cycles, especially when it bears mechanical stresses to adapt to them. The cell cytosol behaves thus like the extracellular matrix; both experience fluidization and gelification.

Hence, the plasma membrane of mastocytes is stretched and sheared. The sensed mechanical stress deforms plasmalemmal molecules. It even actuates a set of proteins embedded in the lipidic bilayer constituting the plasma membrane, the so-called mechanosensitive components, among which some are types of calcium channels. Activated channels enable calcium influx. Calcium ion is a well known cellular messenger that can trigger a set of cascades of chemical reactions as well as various phenomena such as exocytosis of stored materials.

\section{Mechanical stimulation.}

The classical mode of acupuncture relies on the following set of events: (1) generation 
of a local stress field caused by needle rotation and/or translational motions; (2) mechanotransduction of sensed local tension into chemical signals, i.e., increase in cytosolic $\mathrm{Ca}^{2+}$ concentration, granule exocytosis, and substance release; (3) early, quick triggering of action potentials by nerve stimulants bound to their cognate receptors on local nerve terminals and activation of the target brain region that is associated with a local functional hyperemia (local increase in blood flow due to nervous activity); (4) local elevation of vascular permeability and vasodilation primed by released compounds with increased local blood flow by the sent cardiotonic messengers, all these processes enhancing endocrine signaling, i.e., delayed stimulation of the target brain region, as endocrine messengers are preferentially distributed in active brain regions; and (5) chemotaxis of mastocytes from regional pools and circulating blood and degranulation of newly arrived mastocytes at acupoints that enables a sustained process, degranulation being effective only near the needle, where the mechanical stress is high enough.

\section{Method}

In the present paper, the operating mode is needling with initial and periodic needle rotation and/or translational motions (needling with lifting-thrusting cycle or twisting). The resulting local stress field engenders a permanently deformed region, in fact that experienced during acupuncture a ball of about 5-mm caliber centered on the needle tip in the subcutaneous tissue.

\subsection{Biological model and hypotheses}

The acupoint is considered as a high density pool of mastocytes. Two mastocyte states are considered according to the location with respect to the stimulation region around the inserted needle : granulated and degranulated.

Acupuncture effects rely on intra-, auto-, juxta-, para-, and endocrine signaling aimed at triggering mastocyte chemotaxis and sending signals locally to nerve endings and lymph and blood capillaries. Liganded receptors on the nerve surface very rapidly launch action potentials that travel to the central nervous system. Liganded receptors on the vascular cell surface increase the vascular permeability and provoke vasodilation, thereby facilitating the transfer of molecules into the lymph and blood. Once conveyed in blood, messengers target the central nervous system and the cardiac pump to enhance blood flow at acupoints.

A quasi-instantaneous release of chemical mediators is assumed upon stimulation by the stress field resulting from needling and $\mathrm{Ca}^{++}$ion entry into mastocytes. The magnitude orders for $\mathrm{Ca}^{++}$channel activation and $\mathrm{Ca}^{++}$entry into the cytosol from the extracellular milieu are actually $\mathcal{O}(\mathrm{ms})$ and $\mathcal{O}(\mathrm{s})$, respectively.

Once the content stored in intracellular vesicles is liberated, acupuncture can be modeled by an immediate and a late response corresponding to nervous (action potentials) and bloodborne signals. 
Chemotaxis, that is, the recruitment of mastocytes from the local blood circulation and locoregional pools, enables a permanent release of substances during the delayed regeneration of granules content. This mechanism is assumed to be the essential phenomenon of acupuncture that enables the process maintenance.

The extracellular space constitutes a single compartment that consists of a fluid (interstitial fluid) and solid (matrix) fraction (phase). Its composite material is constituted of fibers embedded in the semipermeable ground substance. This porous medium is crossed by the interstitial fluid that, under mechanical stress, flows slowly along fibers and through gaps of the macromolecular meshwork and bundles of elastin and collagen fibers, the viscous dissipation being the predominant term (Darcy-Brinkman flow). The Darcy's law, a phenomenologically derived equation can be obtained from the linear Stokes' equations in a periodic unit cell using the homogeneization theory with a nonpenetration condition at the domain boundary. Matrix conductance is defined as the flow of interstitial fluid per unit pressure drop across unit area; matrix resistance is the inverse of conductance. Moreover, the extracellular matrix evolves between a fluid and a gel, according to local mechanical, physical, and chemical conditions (fluidization-gelification cycle). In the present work, the Stokes-like flow in the extracellular matrix and hence convection in the involved mass transfer process is neglected.

\subsection{Mathematical model}

Any mastocyte in the local vasculature moves along the chemoattractant gradient, hence undergoes a transmigration (across blood vessel wall to exit blood, remaining granulated outside a region of triggering mechanical stress $(x \gg \ell)$ and liberating its granule content, once it reaches a region close to the acupoint $(0-\ell)$, where a significant magnitude of the mechanical stress can be sensed. Similarly, any mastocyte from a neighboring pool can migrate. Two mastocyte states indeed exist according to its localization with respect to the acupoint (non-degranulated and degranulated). Therefore, in the case of mechanotransduction, the mechanical stress is modeled by a compact-supported function.

Chemical mediators are supposed to be quasi-instantaneously released by the mechanotransduction process that mainly relies on a sudden, rapid, and copious calcium entry in the mastocyte cytosol. This calcium wave that gushes and pervades the mastocyte enables it to discharge chemoattractants, nerve messengers, cardiovascular stimulants, and endocrine messengers. On the other hand, the regeneration of granules content inside the mastocyte is delayed and slow.

Following chemotaxis from regional pools and blood, newly arrived mastocytes at acupoints experience a degranulation triggered by the stress field. The resulting selfsustained process enables the local elevation of vascular permeability for improved cardiac output and enhanced endocrine signaling, remote cardiac effect cooperating with vasodilation to raise local blood flow, endocrine signaling to central nervous system being supported by neuronal activity in the brain region of interest (hyperemia), that simultaneously receive sets of action potentials. 
The mathematical model is an initial boundary value problem composed of a set of five partial differential equations for a bounded domain $(x \in \Omega)$ and evolving time $\left(t \in \mathbb{R}^{+}\right)$. Two equations are related to the populations of granulated and degranulated mastocytes. This model of mastocyte response to acupuncture needling is based on the Keller-Segel model for chemotaxis. Three associated equations describe the temporal evolution of concentrations of chemoattractants $(c(t, x))$, liberated nerve stimulants $\left(s_{n}(t, x)\right)$, and endocrine activators $\left(s_{e}(t, x)\right)$ of some sites of the central nervous system in particular:

$$
\begin{aligned}
\partial_{t} n_{g}-\mathcal{D}_{m} \nabla^{2} n_{g}+\nabla \cdot\left(\mathrm{S} n_{g} \nabla c\right) & =-\mathrm{A} \Phi n_{g}+\mathrm{R} n_{d} \\
\partial_{t} n_{d}-\mathcal{D}_{m} \nabla^{2} n_{d} & =\mathrm{A} \Phi n_{g}-\mathrm{R} n_{d} \\
\partial_{t} c-\mathcal{D}_{c} \nabla^{2} c & =\kappa_{c} \mathrm{~A} \Phi n_{g}-\mathrm{D}_{c} c \\
\partial_{t} s_{n}-\mathcal{D}_{n} \nabla^{2} s_{n} & =\kappa_{n} \mathrm{~A} \Phi n_{g}-\mathrm{D}_{n} s_{n} \\
\partial_{t} s_{e}-\mathcal{D}_{e} \nabla^{2} s_{e} & =\kappa_{e} \mathrm{~A} \Phi n_{g}-\mathrm{D}_{e} s_{e}
\end{aligned}
$$

In the above, $n_{g}(t, x)$ and $n_{d}(t, x)$ are the densities of granulated and degranulated mastocytes, respectively, $\Phi(x)$ the dimensionless magnitude of mechanical stress (i.e., bulk magnitude of locally exerted pressure and shear stress; $0 \leq \Phi(x) \leq 1,0 \leq \mathbf{x} \leq \ell$; $\ell$ : space range of the applied stress) in the mechanical stress-responsive region (area in $2 \mathrm{D}$ [volume in $3 \mathrm{D}$ ]); $\mathcal{D}_{m / c / n}$ the diffusion coefficients; A the activation rate of granulated mastocytes subjected to the mechanical stress $\Phi$ that then degranulate; $R$ the regeneration rate of degranulated mastocytes; $S$ the mastocyte sensitivity to chemoattractant; $\mathrm{D}_{c, e, n}$ the degradation rate; and $\kappa_{c, e, n}$ the release quantity coefficient. In these equations, the parameters $S$ and $\kappa_{c, e, n}$ ensure the physical homogeneity. All the parameters $\left(\mathrm{S}, \mathcal{D}_{m / c / n / e}, \mathrm{~A}, \mathrm{R}, \mathrm{D}\right.$, and $\left.\kappa\right)$ are supposed to be constants. Equation variables and parameters are given with their physical units in Table 3.

The system of equations (2.1) is endowed with boundary conditions, that is, homogeneous Neumann boundary conditions:

$$
\mathbf{n} \cdot \nabla n_{d}=0, \quad \mathbf{n} \cdot \nabla n_{g}=0, \quad \mathbf{n} \cdot \nabla c=0, \quad \mathbf{n} \cdot \nabla s_{n}=0, \quad \mathbf{n} \cdot \nabla s_{e}=0,
$$

or zero total flux boundary conditions given by

$$
\mathbf{n} \cdot\left(\nabla n_{g}-\mathrm{S} \nabla c\right)=0, \quad \mathbf{n} \cdot \nabla n_{d}=0, \quad \mathbf{n} \cdot \nabla c=0, \quad \mathbf{n} \cdot \nabla s_{n}=0, \quad \mathbf{n} \cdot \nabla s_{e}=0,
$$

where $\mathbf{n}$ denotes the outward unit normal on the boundary $\Gamma$.

The equation system (2.1) is also endowed with the prescribed initial conditions:

$$
n_{g}(t=0)=n_{g}^{0}, \quad n_{d}(t=0)=n_{d}^{0}, \quad c(t=0)=c^{0}, \quad s_{n}(t=0)=s_{n}^{0}, \quad s_{e}(t=0)=s_{e}^{0} .
$$

In the present model of chemotaxis, the chemoattractant is emitted from a a subdomain centered on the needle in which mechanical stress is sufficiently intense to be detected and transduced by mastocytes. The stress function $\Phi$ can be represented as a $C^{\infty}$ 
Table 3: Equation variables and parameters with physical dimensions (units) expressed in the fundamental MLT (length, mass, time) system.

\begin{tabular}{lll}
\hline Parameter & Definition & Physical dimension \\
\hline & & \\
A & Activation rate of mastocytes & $\mathrm{T}^{-1}$ \\
C & Concentration & $\mathrm{mol}^{-} \mathrm{L}^{-3}$ \\
$\mathrm{D}$ & Degradation rate & $\mathrm{T}^{-1}$ \\
$\mathcal{D}$ & Diffusion coefficient & $\mathrm{L}^{2} \cdot \mathrm{T}^{-1}$ \\
$n$ & Mastocyte density & $\mathrm{L}^{-3}$ \\
$\mathrm{R}$ & Regeneration rate of & $\mathrm{T}^{-1}$ \\
& mastocyte granules & \\
$\mathrm{S}$ & Mastocyte sensitivity & $\mathrm{L}^{5} \cdot \mathrm{mol}^{-1} \cdot \mathrm{T}^{-1}$ \\
& to chemoattractant & \\
$\kappa$ & Release quantity coefficient & mol \\
$\Phi$ & Mechanical stress function & Dimensionless \\
\hline
\end{tabular}

and compactly supported function from $\mathbb{R}^{2}$ to $[0,1]$ with the following properties:

$$
\begin{aligned}
& \forall(x, y) \in \mathbb{R}^{2},|\Phi(x)-\Phi(y)| \leq k_{\Phi}|x-y| \\
& \Phi(x) \leq 1, \forall x \in \mathbb{R}^{2}, \\
& \Phi(x)=0, \forall x \in \mathbb{R}^{2},|x| \geq \ell .
\end{aligned}
$$

In the numerical simulations, it is represented by a bump function using two positive parameters $\alpha$ and $\beta$ that control amplitude and shape:

$$
\Phi_{B}(x, y)=\left\{\begin{array}{l}
\alpha \exp \left(-\beta /\left(\ell^{2}-x^{2}-y^{2}\right)\right), \text { if } x^{2}+y^{2}<\ell^{2} \\
0, \text { elsewhere }
\end{array}\right.
$$

Distribution analysis relies on statistical moments (mean, variance, skewness, and kurtosis, among others). The initial mastocyte distribution is represented by a normal (Gaussian) distribution:

$$
n(x, y, t=0)=a \exp \left[-\left(\left(x-x_{\max }\right)^{2} / 2 \sigma_{x}^{2}+\left(y-y_{\max }\right)^{2} / 2 \sigma_{y}^{2}\right)\right]+b, \quad(x, y) \in \mathbb{R}^{2},
$$

where $a$ is the mastocyte distribution amplitude, $b$ the minimal mastocyte density in the tissue, $\sigma_{x}$ and $\sigma_{y}$ the dispersions in the $x$ - and y-direction, and $\left\{\left(x_{\max }, y_{\max }\right)\right\}$ the coordinates of the site of maximal mastocyte density. When $b_{n}=0, \sigma_{x}=\sigma_{y}=\sigma$, and $x_{\max }=y_{\max }=0$, the number of mastocyte is given by

$$
m_{\left.\right|_{\mathbb{R}^{2}}}=\int_{-\infty}^{-\infty} \int_{-\infty}^{-\infty} n(x, y, t=0) d x d y=2 \pi a \sigma^{2} .
$$


In the disc of center $(0,0)$ with radius $R$, the number of mastocyte is given by

$$
\begin{aligned}
m_{\left.\right|_{D(0, R)}} & =\iint_{D(0, R)} n(x, y, t=0) d x d y \\
& =\int_{0}^{2 \pi} \int_{0}^{R} a \operatorname{Rexp}\left[-\left(R^{2} / 2 \sigma^{2}\right)\right] d r d \theta \\
& =2 \pi a \sigma^{2}\left(1-e^{-\left[R^{2} / 2 \sigma^{2}\right]}\right) .
\end{aligned}
$$

The spatial distribution of mastocytes is described by the zeroth and second statistical moments:

$$
m_{0}(t):=\int_{\mathbb{R}^{2}} n_{g}(t, x) d x ; \quad m_{2}(t):=\int_{\mathbb{R}^{2}} \frac{|x|^{2}}{2} n_{g}(t, x) d x .
$$

A small second moment indicates that the cells are gathered in the vicinity of the center of the domain.

A simplest form of the equation set describes the evolution of the density of mastocytes and the chemoattractant concentration, assuming that the diffusion of the chemoattractant is instantaneous and hence implying the absence of evolving (in time) chemoattractant concentration:

$$
\begin{aligned}
\partial_{t} n_{g}-\mathcal{D}_{m} \nabla^{2} n_{g}+\nabla \cdot\left(\mathrm{S} n_{g} \nabla c\right) & =-\mathrm{A} \Phi n_{g}, \quad t>0, x \in \Omega, \\
\mathcal{D}_{c} \nabla^{2} c & =-\kappa_{c} \mathrm{~A} \Phi n_{g}, \\
\left.n_{g}\right|_{t=0} & =n_{g}^{0} .
\end{aligned}
$$

In the differential system (2.11), the total mass is conserved, that is, the total number of cells in the domain remains constant, but their localization changes:

A singularity appears in a finite time and the cell density blows up at the singularity point [11], that is blow-up solution happens, if: (1) the initial density of mastocytes is high enough

$$
m_{0}(0)=\int_{\mathbb{R}^{2}} n_{g}^{0}(x) d x>\frac{8 \pi}{\mathrm{AS} \kappa_{c}} \exp ^{\mathrm{AT}}
$$

and (2) the second moment is small enough (small dispersion)

$$
m_{2}(0)=\int_{\mathbb{R}^{2}}\left|x^{2}\right| n_{g}^{0}(x) d x<\xi^{*}
$$

where

$$
\xi^{*}=\frac{\left(b^{2}-2 a c\right)-b \sqrt{4 a c+b^{2}}}{2 c^{2}}
$$

and

$$
a=2 m_{0}(0)\left(1-\frac{\mathrm{AS} \kappa_{c}}{8 \pi} m_{0}(0) \exp ^{-L T^{*}}\right), \quad b=\frac{3 \sqrt{2}}{2 \pi} \frac{\mathrm{AS} \kappa_{c}}{\lambda} m_{0}(0)^{\frac{3}{2}} \text { and } \quad c=\frac{1}{\pi} \frac{\mathrm{AS} \kappa_{c}}{\lambda} k_{\phi} m_{0}(0) .
$$


In mathematical physics, partial differential equations most often are rendered dimensionless. Dimensionless independent $(\tilde{x}$, and $\tilde{t})$ and dependent variables $\left(\tilde{n}_{g}, \tilde{n}_{d}, \tilde{c}\right.$, $\tilde{s}_{n}$, and $\tilde{s}_{e}$ ) are hence introduced:

$$
\begin{array}{llll}
\tilde{x}=x / \ell ; & \tilde{t}=t \times \mathcal{D}_{m} / \ell^{2} ; & \tilde{n}_{g}=n_{g} / n_{\text {ref }} ; & \tilde{n}_{d}=n_{d} / n_{\text {ref }} ; \\
\tilde{c}=c / c_{\text {ref }} ; & \tilde{s}_{n}=s_{n} / s_{\text {ref }} ; & \tilde{s}_{e}=s_{e} / s_{\text {ref }} . &
\end{array}
$$

The reference quantities are the initial density of granulated mastocytes $\left(n_{\text {ref }}\right)$, concentration of messengers stored before degranulation ( $c_{\text {ref }}$ and $\left.s_{\text {ref }}\right)$.

The set of equations (2.1) then becomes, removing for the sake of simplificity the tilde denoting dimensionless variables:

$$
\begin{aligned}
\partial_{t} n_{g}-\nabla^{2} n_{g}+\Pi_{\mathrm{S}} \nabla \cdot\left(n_{g} \nabla c\right) & =-\Pi_{\mathrm{A}} \Phi n_{g}+\Pi_{\mathrm{R}} n_{d} \\
\partial_{t} c-\Pi_{\mathcal{D}_{c}} \nabla^{2} c & =\Pi_{\mathcal{K}_{c}} \Phi n_{g}-\Pi_{\mathrm{D}_{c}} c \\
\partial_{t} n_{d}-\nabla^{2} n_{d} & =\Pi_{\mathrm{A}} \Phi n_{g}-\Pi_{\mathrm{R}} n_{d} \\
\partial_{t} s_{n}-\Pi_{\mathcal{D}_{n}} \nabla^{2} s_{n} & =\Pi_{\mathcal{K}_{n}} \Phi n_{g}-\Pi_{\mathrm{D}_{n}} s_{n} \\
\partial_{t} s_{e}-\Pi_{\mathcal{D}_{e}} \nabla^{2} s_{e} & =\Pi_{\mathcal{K}_{e}} \Phi n_{g}-\Pi_{\mathrm{D}_{e}} s_{e}
\end{aligned}
$$

where

$$
\begin{array}{lcc}
\Pi_{\mathrm{S}}=\frac{S c_{\mathrm{ref}}}{\mathcal{D}_{m}} ; & \Pi_{\mathrm{A}}=\frac{\ell^{2} \mathrm{~A}}{\mathcal{D}_{m}} ; & \Pi_{\mathrm{R}}=\frac{\ell^{2} \mathrm{R}}{\mathcal{D}_{m}} ; \\
\Pi_{\mathcal{D}_{c}}=\frac{\mathcal{D}_{c}}{\mathcal{D}_{m}} ; & \Pi_{\mathcal{K}_{c}}=\frac{\kappa_{c} \mathrm{~A} \ell^{2} n_{\mathrm{ref}}}{\mathcal{D}_{m} c_{\mathrm{ref}}} ; & \Pi_{\mathrm{D}_{c}}=\frac{\ell^{2} \mathrm{D}_{c}}{\mathcal{D}_{m}} ; \\
\Pi_{\mathcal{D}_{n}}=\frac{\mathcal{D}_{n}}{\mathcal{D}_{m}} ; & \Pi_{\kappa_{n}}=\frac{\kappa_{n} \mathrm{~A} \ell^{2} n_{\mathrm{ref}}}{\mathcal{D}_{m} S_{\mathrm{ref}}} ; & \Pi_{\mathrm{D}_{n}}=\frac{\ell^{2} \mathrm{D}_{n}}{\mathcal{D}_{m}} ; \\
\Pi_{\mathcal{D}_{e}}=\frac{\mathcal{D}_{e}}{\mathcal{D}_{m}} ; & \Pi_{\mathcal{K}_{e}}=\frac{\kappa_{e} \mathrm{~A} \ell^{2} n_{\mathrm{ref}}}{\mathcal{D}_{m} S_{\mathrm{ref}}} ; & \Pi_{\mathrm{D}_{e}}=\frac{\ell^{2} \mathrm{D}_{e}}{\mathcal{D}_{m}} .
\end{array}
$$

\subsection{Solving procedure}

Numerical simulations were carried out using the FreeFem ++ software, an open source partial differential equation solver based on the variational formulation using the $\mathrm{C}++$ language code developed in the Laboratory Jacques-Louis Lions (LJLL) of University Pierre et Marie Curie (UPMC, Paris, France) [10]. The FreeFem ++ software comprises the following modulus: triangular finite element spaces; 2D and 3D automatic mesh generator and mesh adaptation; fast linear solvers; and MPI tools for parallel computing. Neumann boundary conditions can be directly implemented into the weak formulation without introducing any derivation error. A penalty method is employed to impose Dirichlet boundary conditions. To solve the linear system associated to the discretized problem, FreeFem ++ provides many direct and iterative solvers. In the present study, the multifrontal solver UMFPACK and parallel solver MUMPS are utilized.

As in the FreeFem ++ software, problems are expressed in the weak formulation, equations are multiplied by a test function $w_{i}$ in $\left.H^{1}(\Omega)\right)^{5}(i=g, d, c, n, e)$ and integrated 
over the domain $\Omega$ ). In the presence of a nonlinear term, the equation is artificially decoupled using the previously computed solutions. The solving procedure relies on a semi-discretization in time. Mesh adaptativity enables to follow the local evolution of the solution. The algorithm for the decoupled method is given as follows

1. Find $c^{m+1} \in V_{c}^{h}$ such that

$$
\int_{\Omega} \frac{c^{m+1}-c^{m}}{\Delta t} w_{c}+\int_{\Omega} \mathcal{D}_{c} \nabla c^{m+1} \nabla w_{c}+\int_{\Omega} \mathrm{D}_{c} c^{m+1} w_{c}=\int_{\Omega} \kappa_{c} \Phi n_{g}^{m} w_{c}, \quad \forall w_{c} \in V_{c}^{h} .
$$

2. Find $n_{d}^{m+1} \in V_{d}^{h}$ such that

$$
\int_{\Omega} \frac{n_{d}^{m+1}-n_{d}^{n}}{\Delta t} w_{d}+\int_{\Omega} \nabla n_{d}^{m+1} \cdot \nabla w_{d}+\int_{\Omega} k_{r} n_{d}^{m+1} w_{d}=\int_{\Omega} \mathrm{A} \Phi n_{g}^{m} w_{d}, \quad \forall w_{d} \in V_{d}^{h} .
$$

3. Find $n_{g}^{m+1} \in V_{g}^{h}$ such that

$$
\begin{array}{r}
\int_{\Omega} \frac{n_{g}^{m+1}-n_{g}^{n}}{\Delta t} w_{g}+\int_{\Omega} \nabla n_{g}^{m+1} \cdot \nabla w_{g}-\int_{\Omega} \mathrm{S} n_{g}^{m+1} \nabla c^{m+1} \cdot \nabla w_{g}+\int_{\Omega} \mathrm{A} \Phi n_{g}^{m+1} w_{g} \\
=\int_{\Omega} k_{r} n_{d}^{m+1} w_{g}, \quad \forall w_{g} \in V_{g}^{h} .
\end{array}
$$

4. Find $s_{n}^{m+1} \in V_{n}^{h}$ such that

$$
\begin{aligned}
\int_{\Omega} \frac{s_{n}^{m+1}-s_{n}^{m}}{\Delta t} w_{n}+\int_{\Omega} \mathcal{D}_{s_{n}} \nabla s_{n}^{m+1} \cdot \nabla w_{n}+\int_{\Omega} \mathrm{D}_{n} s_{n}^{m+1} w_{n}= & \int_{\Omega} \kappa_{n} \Phi n_{g}^{m+1} w_{n}, \\
& \forall w_{n} \in V_{n}^{h} .
\end{aligned}
$$

5. Find $s_{e}^{m+1} \in V_{e}^{h}$ such that

$$
\begin{aligned}
\int_{\Omega} \frac{s_{e}^{m+1}-s_{e}^{m}}{\Delta t} w_{e}+\int_{\Omega} \mathcal{D}_{s_{e}} \nabla s_{e}^{m+1} \cdot \nabla w_{e}+\int_{\Omega} \mathrm{D}_{e} s_{e}^{m+1} w_{e}= & \int_{\Omega} \kappa_{e} \Phi n_{g}^{m+1} w_{e}, \\
& \forall w_{e} \in V_{e}^{h} .
\end{aligned}
$$

where $V_{c}^{h}, V_{d}^{h}, V_{g}^{h}, V_{h}^{h}$, and $V_{e}^{h}$ are included in $H^{1}(\Omega)$ and correspond to the discretized finite element spaces for all variables.

The choice of the classical $P^{1}$ finite element spaces for $n_{g}^{m+1}, n_{d}^{m+1}, s_{n}^{m+1}$, and $s_{e}^{m+1}$ is reasonable. However, for $c^{n+1}$, the classical $P^{2}$ finite element space is required. Neumann boundary conditions (no cell and chemoattractant fluxes) are applied at the border of the computational domain. 


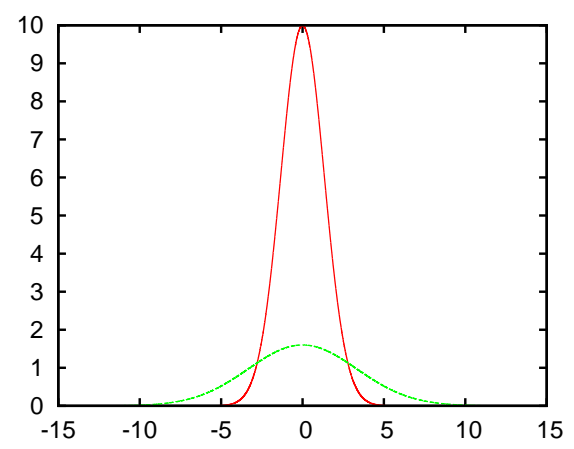

Figure 1: Initial mastocyte Gaussian distribution in an acupoint (concentrated distribution) and non-acupoint (dispersed distribution) with the same cell number.

The solution depends on the chemotactic sensitivity $S$, activation rate $A$, and release coefficient $\kappa_{c}$. To the best of our knowledge, no information on the previous three parameters is available. From the analysis of the model and the two conditions on the total initial mass (2.12) and the initial second moment (2.13), the set of dimensionless parameters is chosen to enlighten the blow-up conditions studied theoretically. For the numerical simulation, the constant parameters are set as follows :

$$
\begin{array}{ccc}
\Pi_{S}=1, & \Pi_{A}=10, & \Pi_{R}=0.001, \\
\Pi_{\mathcal{D}_{c}}=\prod_{\mathcal{D}_{n}}=\Pi_{\mathcal{D}_{e}}=5, & \Pi_{\mathcal{K}_{c}}=\prod_{\mathcal{K}_{n}}=\Pi_{\mathcal{K}_{s}}=1, & \Pi_{\mathrm{D}_{c}}=\prod_{\mathrm{D}_{n}}=\Pi_{\mathrm{D}_{e}}=1 .
\end{array}
$$

\section{Results}

Any acupuncture is characterized by the distribution of mastocytes, the major tissular constituent assumed to be involved in acupuncture. A small second moment of the mastocyte distribution at acupoint means that the mastocyte density is high enough to be responsive to acupuncture stimulation.

Numerical results are presented for two initial mastocyte Gaussian distributions with an identical cell number: (1) acupoint (concentrated distribution; $m_{0}(0)=50, m_{2}(0)=11.12$ ) and (2) nonacupoint (dispersed distribution; $m_{0}(0)=50, m_{2}(0)=165.19$; Fig. 1). The locations of acupoint and non-acupoint are centered on the point of coordinate $(0,0)$ that also corresponds to the point where the needle inserts. The expected blow-up solution is obtained or not (Fig. 2) according to whether the initial distribution of the mastocyte distribution is sharp or blunt (Fig. 1).

The expected blow-up solution is obtained or not relies on whether the needle is inserted far or close to the location of the peak cell density or outside the mastocyte pool. In figure 3 , the needle is inserted at $(0,0)$ while the acupoint is centered at $(-7.5,-7.5)$.

Therefore, a successful acupuncture targets a subcutaneous region where mastocytes densely aggregate. Conversely, acupuncture fails when the needle is implanted in a zone enriched in mastocytes, but these cells spread over a too large volume. In other words, 

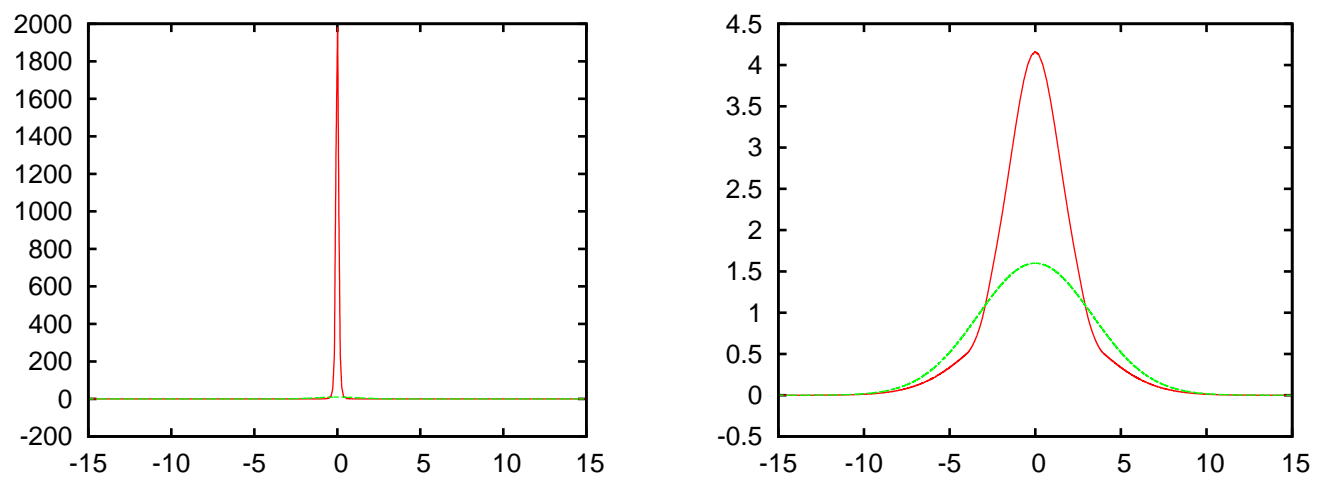

Figure 2: Initial and final distribution of mastocytes at acupoint (left) and in a non-acupoint mastocyte pool (right), three order of magnitude differentiating the ordinate axis of these two plots. Expected blow-up solution is only achieved at acupoint.
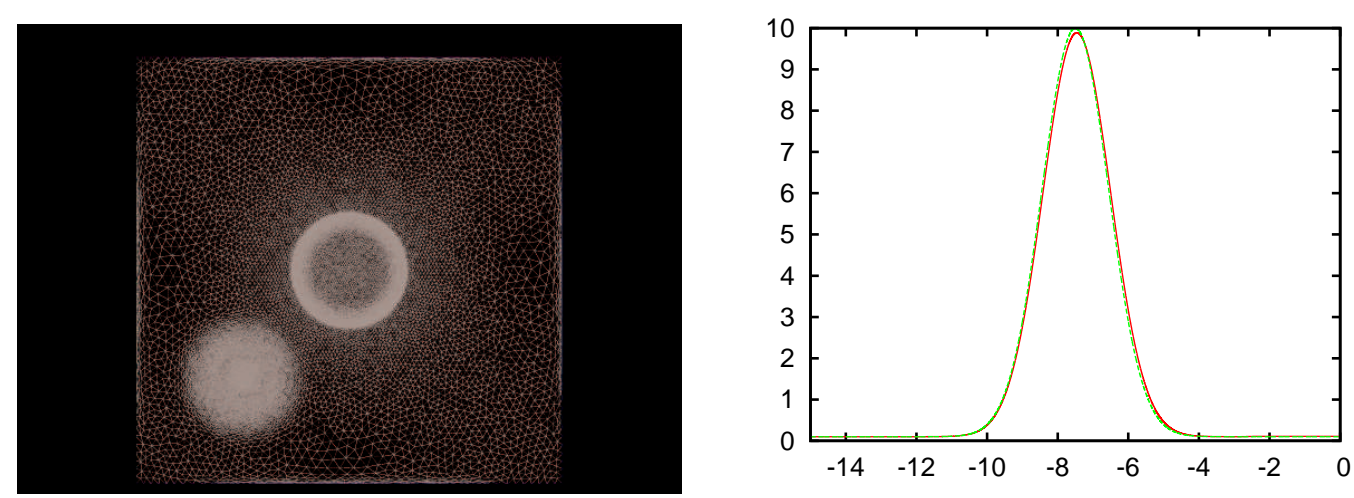

Figure 3: Needling outside an acupoint. Mesh with refinements in the needle region (center) and mastocyte pool (left bottom corner). Absence of significant change in cell population distribution.

blow-up of the solution (i.e., the solution does not remain bounded) occurs in finite time when the initial density of mastocytes around the needle is high enough. Acupuncture is successful when the needle is implanted in the acupoint and not in a nearby pool of mastocytes that can have the same cell number, but in a greater volume (i.e., less dense mastocyte population). Blow-up solution thus results from a strong aggregation of mastocytes and indicates the expertise of the practician and efficiency of the needle manipulation at the selected acupoint.

\section{Discussion}

The present work is aimed at investigating the local effect of acupuncture needling at a single acupoint, but neither effect on the brain and its response to a given target or- 
gan, nor at multiple acupoints. Hence, computations are carried out using the simplified model (2.11).

Mesh size and convergence.

Seven mesh densities (spatial discretization length range 0.004-0.3) using three types of finite elements were tested. In the case of a P1-P2 finite element, the slopes for the mastocyte density $n_{g}$ and concentration $c$ are approximately 2.50, 2.11, and 1.01 and 2.11, 2.10, and 2.08 in the $L^{2}(\Omega), L^{\infty}(\Omega)$, and $H^{1}(\Omega)$ norm, respectively. In the case of a P2P2 finite element, the slopes for the variables $n_{g}$ and $c$ are about 2.57, 2.70, and 2.37 and 2.06, 2.07, and 2.07 in the $L^{2}(\Omega), L^{\infty}(\Omega)$, and $H^{1}(\Omega)$ norm, respectively. The rate of convergence for $n_{g}$ in the $H^{1}(\Omega)$ norm is highly improved. In the case of a P2-P3 finite element, the rates of convergence for $n_{g}$ and $c$ are not enhanced.

\section{Sensitivity to the model parameters.}

The quality of the solutions of the simplified form of the set of equations depends on the chemotactic sensitivity $(\mathrm{S})$, the activation rate $(\mathrm{A})$, and the release coefficient $\left(\kappa_{c}\right)$. Numerical simulations were carried out using a P2-P2 finite element and three mesh sizes $(1 / 16,1 / 64$, and $1 / 256)$ to check the sensitivity of the computation to the chemotactic sensitivity parameter (range 1-100). The chemotactic sensitivity parameter affects strongly the solution of the mastocyte density $n_{g}$ and very slightly that of the concentration $c$. Therefore, mesh refinement is mandatory. In addition, mesh adaptation allows to refine the mesh locally according to the weight of the chemotaxis term $\mathrm{S} \nabla \cdot\left(n_{g} \nabla c\right)$.

\section{Concluding remarks}

During acupuncture, the chemotaxis maintains the transmission of proper messengers to the organs of interest, primarily the brain. A blow-up occurs only when the initial number of mastocytes is high enough and when mastocytes are strongly concentrated at the acupoint, that is, they form a very dense cluster. A blow-up solution of the chemotaxis problem means that incoming mastocytes converge toward the needle, the expected behavior.

According to the initial distribution of the mastocyte distribution, that is, a true acupoint or an inappropriate nearby mastocyte population, and to the needle position with respect to the location of the maximum of the mastocyte distribution, that is, according to whether the practician is an expert or not, the expected blow-up solution is obtained or not.

\section{Acknowledgment}

This study was partly supported by Foundation Sciences Mathématiques de Paris (FSMP). Y.D. received a PhD. grant from Pierre and Marie Curie University - Sorbonne University and was also supported by CASTS at the National Taiwan University. 


\section{References}

[1] Deleuze Y, Thiriet M, TWH Sheu (2015) Modeling and simulation of local physical stress on mastocytes created by the needle manipulation during acupuncture. Communications in Computational Physics (submitted)

[2] Thiriet M (2011) Cell and Tissue Organization in the Circulatory and Ventilatory Systems, Series Biomathematical and Biomechanical Modeling of the Circulatory and Ventilatory Systems (Vol. 1), Springer, New York

[3] Zhou F, Huang D, Xia Y (2010) Neuroanatomical basis of acupuncture points (Chap. 2; 32-80), In Xia Y, Cao X, Wu G, Cheng J (Eds.) Acupuncture Therapy for Neurological Diseases: A Neurobiological View, Tsinghua University Press, Beijing, China and Springer, Berlin Heidelberg

[4] Thiriet M (2013) Tissue Functioning and Remodeling in the Circulatory and Ventilatory Systems, Series Biomathematical and Biomechanical Modeling of the Circulatory and Ventilatory Systems (Vol. 5), Springer, New York

[5] Zhang ZJ, Wang XM, McAlonan GM (2012) Neural acupuncture unit: a new concept for interpreting effects and mechanisms of acupuncture. Evidence-Based Complementary and Alternative Medicine 2012 (2012): 1-23.

[6] Thiriet M (2012) Intracellular Signaling Mediators in the Circulatory and Ventilatory Systems. Series Biomathematical and Biomechanical Modeling of the Circulatory and Ventilatory Systems (Vol. 4), Springer, New York

[7] Zhang D, Ding G, Shen X, Yao W, Zhang Z, Zhang Y, Lin J, Gu Q (2008) Role of mast cells in acupuncture effect: a pilot study. Explore (New York) 4:170-177

[8] Wei F, Shi X, Chen J, Zhou L (2012) Fluid shear stress-induced cytosolic calcium signaling and degranulation dynamics in mast cells. Cell Biology International Reports 19, no. 2 (January 1, 2012): 45-51.

[9] Yao W, Li Y, Ding G (2012) Interstitial fluid flow: The mechanical environment of cells and foundation of meridians. Evidence-Based Complementary and Alternative Medicine 2012:19

[10] Hecht F (2013) New Development in FreeFem ++. Journal of Numerical Mathematics 20, no. 3-4 (2013): 251.

[11] Deleuze Y (2013) A mathematical model of mast cell response to acupuncture needling. Comptes Rendus Mathematique 351, no. 3-4 (2013): 101-5. 